\title{
Design of a Close-Up Stereo Vision Based Sheet Metal Inspection System
}

\author{
M.S. Goldstein \\ J.P. Mitchell \\ R.V. Fleisig \\ A.D. Spence \\ Mechanical \\ Electrical and \\ Mechanical \\ Mechanical \\ Engineering \\ Computer Engineering \\ Engineering \\ Engineering \\ McMaster University McMaster University McMaster University \\ McMaster University \\ goldstms@mcmaster.camitchejp@mcmaster.carobert@mcmaster.ca adspence@mcmaster.ca
}

\begin{abstract}
This paper describes the design and implementation of a close-up stereo computer vision based sheet metal strain and geometry inspection system. A square grid pattern is first applied to the part using either electrochemical etching or screen printing. The part is formed, and measured using a two camera sensor that can be attached to a CMM or FARO arm. Image processing is used to extract the corners of the deformed grid for either three dimensional reconstruction, or for strain analysis. Graphical results are conveniently displayed using HOOPS. Sample measurements using both steel and aluminum test domes indicate an achievable accuracy comparable to other systems.
\end{abstract}

\section{Introduction}

Sheet metal forming is an important manufacturing process used for automobile body and aircraft component production as well as for household washers, dryers and refrigerators. To ensure part quality, inspection to verify adherence to both strain and geometry criteria is important. Conventionally, these two criteria are separately measured, and results are therefore difficult to correlate.

Laboratory strain testing is frequently performed using test domes. Prior to forming, a grid is applied to the sheet metal surface. The dome is then formed and the grid distortion measured. If only strain information is required a transparent tape or handheld CCD camera system can be used. Geometry information requires a tabletop system or equivalent. Conventionally, geometry for larger manufactured parts is measured by taking the part to an isolated Coordinate Measuring Machine (CMM) room and using a touch trigger probe, but strain measurement using this method is inconvenient. For press shop floor use, a preferred solution is to use a portable
FARO arm, but close-up stereo vision is required to achieve acceptable accuracy.

Design of such a close-up stereo vision sensor and sheet metal inspection system is the topic of this paper. The sensor was designed for use on either a CMM or a FARO arm, and has an accuracy comparable to other less agile systems. Measured results are conveniently displayed using Forming Limit Diagrams (FLDs) and colour 3-D thickness strain plots. HOOPS graphics provides platform portability and supports visualization using ordinary displays as well as 3-D shutter glasses and anaglyphs.

The remainder of the paper is organized as follows. Section 2 presents technical background material on strain measurement and analysis. Section 3 describes the stereo vision sensor design. Section 4 details the image processing algorithms. Experimental results obtained with the system are presented in Section 5. Section 6 discusses the achievements to date, and suggests avenues for continuing investigation. Conclusions are presented in Section 7.

\section{Strain Measurement and Analysis}

The strain characteristics of a particular material are conveniently represented using a Forming Limit Diagram (FLD) (Figure 1) [1] that is obtained from the supplier. Major/minor strains in Zone C, above the Forming Limit Curve (FLC) are unacceptable, and will result in tearing or wrinkling. Zone $\mathbf{B}$ is marginal. Normal production requires that the part strains remain in the safe Zone, $\mathbf{A}$.

Typically, experimental strain measurement begins with electrochemical etching or screen printing of a regular circle or square grid on the undeformed sheet metal. The part is then formed into a test dome (Figure 2), and the distorted grid is measured. For circle grids, direct measurement can be made using a graduated, transparent Mylar $\AA$ tape (Figure 3) [2] or a hand held CCD camera and associated software (Figure 4) [3]. Simultaneous geometry measurement 


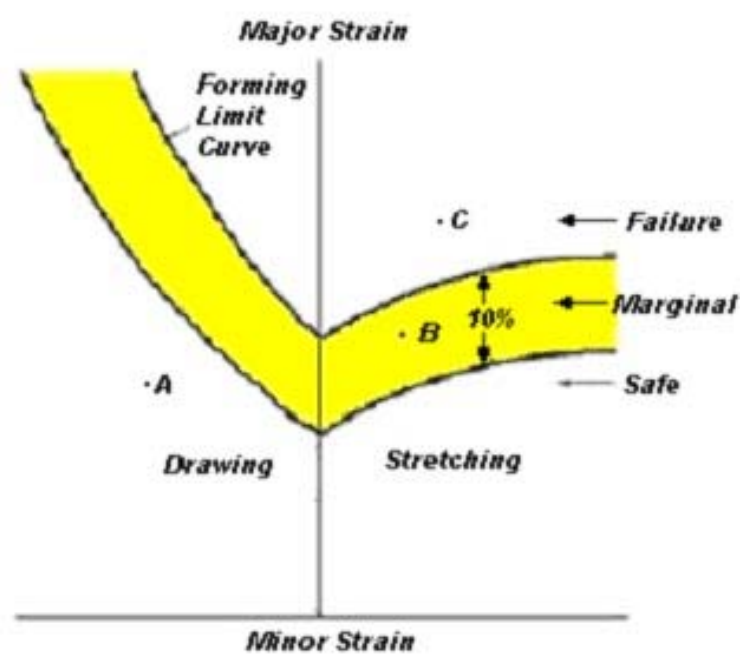

Figure 1. Forming Limit Diagram (FLD) [1]

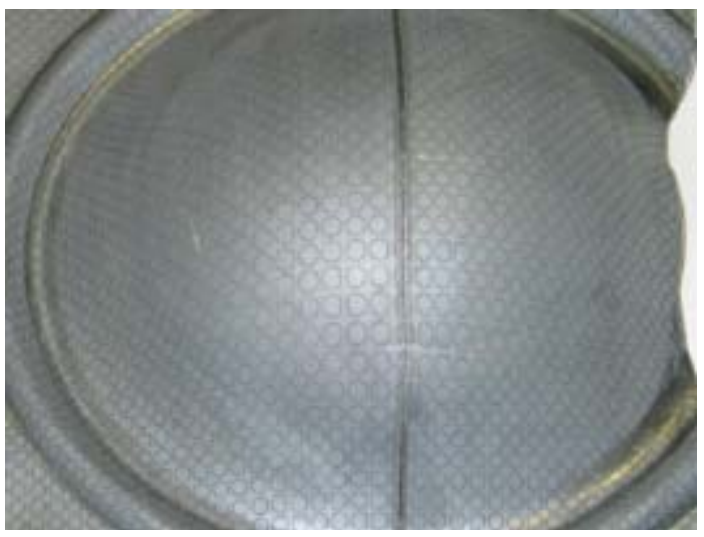

Figure 2. Formed tailor welded steel test dome with circle grid. Undeformed left side thickness: $0.64 \mathrm{~mm}$, right side $1.14 \mathrm{~mm}$.

requires a tabletop system (Figure 5) [4] consisting of a rotary table and fixed camera. The dome is imaged from several viewpoints to reconstruct the 3-D geometry.

For larger manufactured parts, geometry is measured using a touch trigger probe equipped Coordinate Measuring Machine (CMM) located in an isolated room. More rapid geometry measurement is possible using active laser triangulation line digitizers (Figure 6), and extension to use the digitizer gray level signal has previously been reported [5]. For example, for the tailor welded steel dome (Figure 2), the major $\left(\varepsilon_{1}\right)$ and minor $\left(\varepsilon_{2}\right)$ strain FLD (Figure 7(a)) was computed using the major/minor true strain equations,

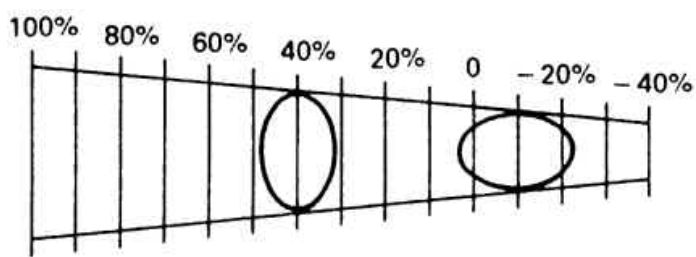

Figure 3. Transparent Mylar ${ }^{\circledR}$ tape strain measurement [2]

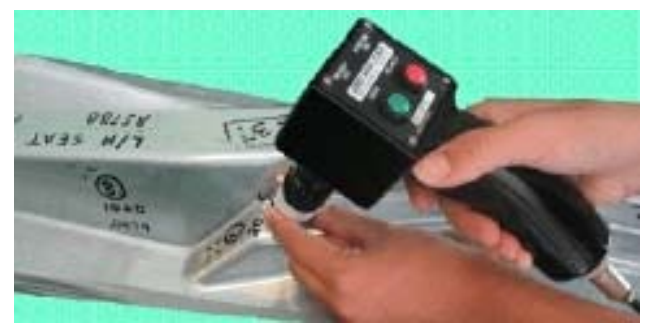

Figure 4. Hand held strain measuring camera [3]

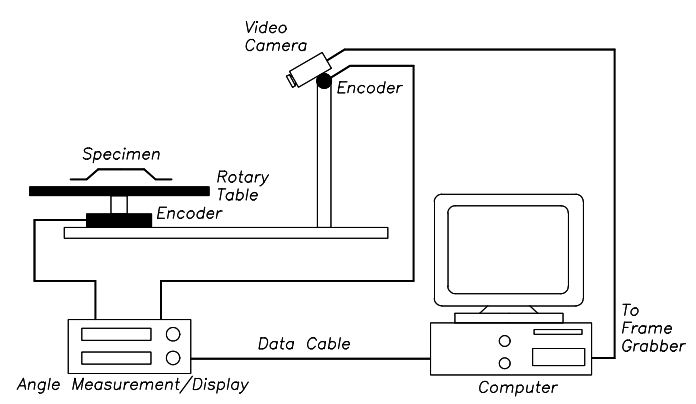

Figure 5. Tabletop strain measuring system [4]

$$
\varepsilon_{1}=\int_{L_{0}}^{L_{1}} \frac{d L}{L}=\ln \left(\frac{L_{1}}{L_{0}}\right) ; \quad \varepsilon_{2}=\int_{L_{0}}^{L_{2}} \frac{d L}{L}=\ln \left(\frac{L_{2}}{L_{0}}\right)
$$

where $L_{1}$ is the ellipse major axis length, $L_{2}$ is the minor axis length, and $L_{0}$ is the undeformed circle diameter. Based on conservation of material volume, the thickness strain is simply computed using $\varepsilon_{3}=-\varepsilon_{1}-\varepsilon_{2}$ (Figure 7(b)). Laser line digitizers have also been mounted on portable CMMs such as the convenient FARO arm (Figure 8) [6]. This solution is acceptable for geometry measurement, but does not provide the high resolution, uniform sample point spacing required for strain analysis. As well, current sensor units are too heavy for an operator to hold for long time periods.

A more agile alternative for stamping press shop floor use is close-up computer stereo vision. 


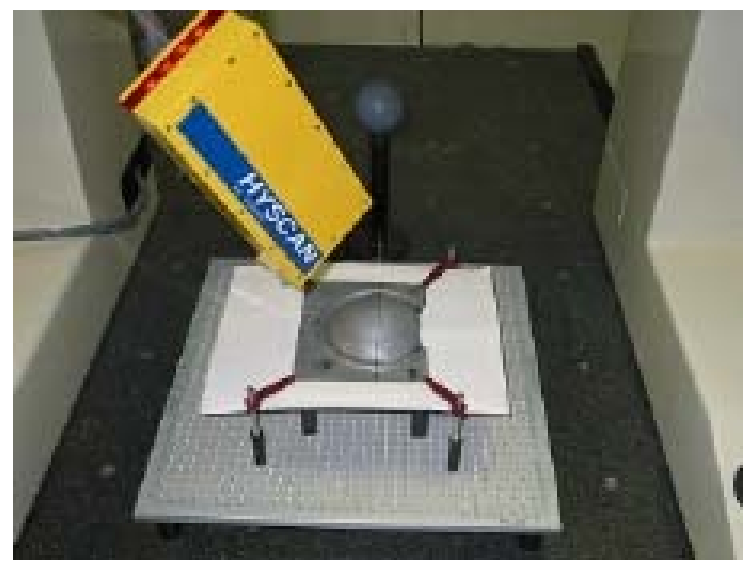

Figure 6. Laser digitizer/CMM based simultaneous strain/geometry measurement [5]

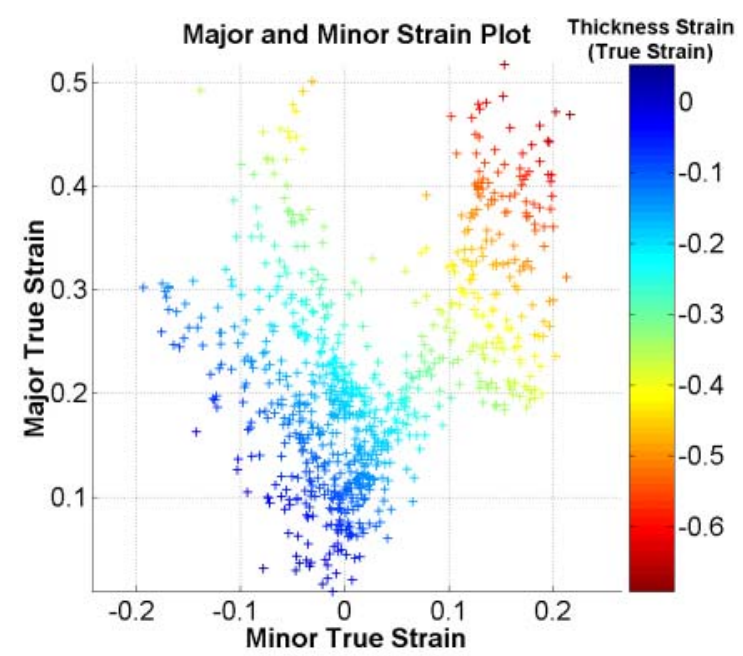

(a) Forming Limit Diagram (FLD)

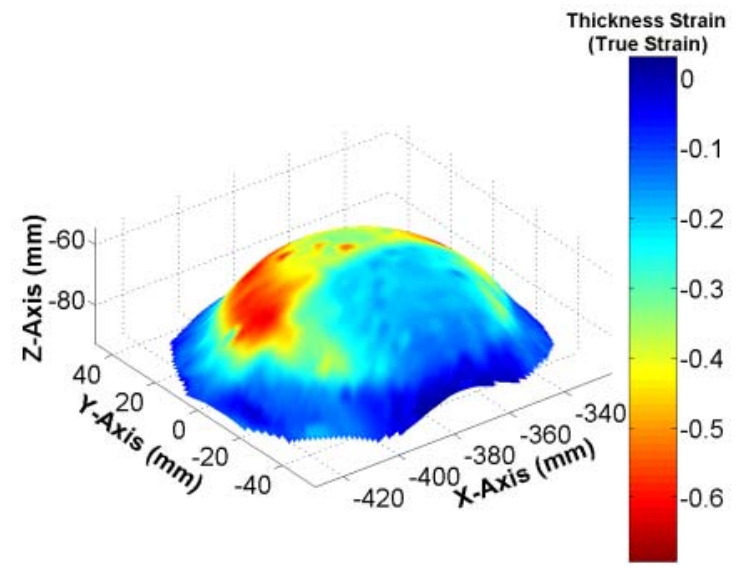

(b) 3-D thickness strain

Figure 7. Strain plots for steel test dome (Figure 2) [5]

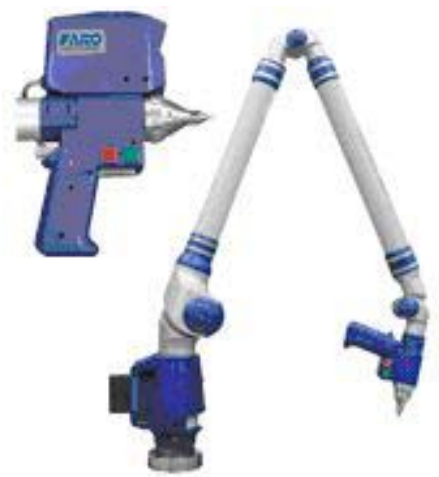

Figure 8. FARO portable arm CMM and laser digitizer [6]

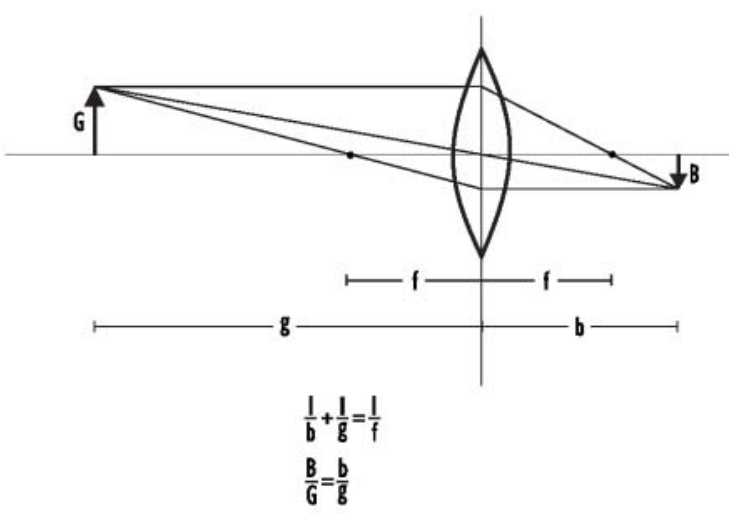

Figure 9. Lens law variables [8]

\section{Sensor Design}

Computer stereo vision is a well researched topic. The basic concept is to image a scene from two (or more) viewpoints, extract features, match features common to multiple viewpoints, and calculate the 3-D position. A familiar geographical application is photogrammetry, in which aerial photographs are processed to produce topographical maps.

The sheet metal application considered herein requires a much finer resolution, and hence close-up stereo vision was used. A typical grid size is 2.54 $\mathrm{mm}$, and, for high CMM measurement accuracy, it is desirable to measure strain to approximately 0.1 percent, requiring (after anticipated subpixel accuracy correction) $0.003 \mathrm{~mm}$ resolution. Affordable CCD cameras have a $1024 \times 768$ resolution, resulting in a $32 \mathrm{~mm} \times 24 \mathrm{~mm}$ image area. For the Point Grey IEEE 1394 Dragonfly 8 bit grey level CCD cameras used [7], the square (diagonal) pixel pitch is $0.00465 \mathrm{~mm}$ $(0.00658 \mathrm{~mm})$ - the diameter of the blur spot $u^{\prime}$ [8]. Using a $f=12 \mathrm{~mm}$ focal length Cosmicar/Pentax 
lens, it was experimentally found that, in order to have sufficient operating light, an F-stop of $k=4$ was required. The unmodified Minimum Object Distance (MOD) for the lens is $g=200 \mathrm{~mm}$, and hence the in focus near and far field limits, found from (Figure 9), are respectively:

$$
|g|_{v}=\frac{|g|}{1+u^{\prime} k \frac{|g|-f}{f^{2}}} ;|g|_{h}=\frac{|g|}{1-u^{\prime} k \frac{|g|-f}{f^{2}}}
$$

Substituting actual values yields $|g|_{v}=193 \mathrm{~mm}$ and $|g|_{h}=207 \mathrm{~mm}$. To reduce the MOD, a $1 \mathrm{~mm}$ macro (spacer) ring was added, changing the field limits to $|g|_{v}=115 \mathrm{~mm}$ and $|g|_{h}=125 \mathrm{~mm}$. These are acceptable values for a CMM mounted sensor.

Figure 10 (a) shows a sketch of the stereo vision sensor with outer dimensions labeled. The stereo vision head is roughly $136 \mathrm{~mm}$ by $79 \mathrm{~mm}$, without lenses, when the lenses are at an angle of 20 degrees with the vertical. The assembled unit is seen in Figure 10 (b).

Initially the stereo vision sensor was designed to be used on a CMM with a Renishaw PH10M motorized probe head system, connecting via Renishaw's PAA1 Autojoint to M8 adaptor. The Autojoint system allowed for simple and repeatable connection of the sensor to the CMM. The PH10 is designed to be used with touch trigger probes, and as such is able to relay an electrical signal from the probe head to the CCM to coordinate point capture with scale recording electronics. The sensor design incorporated electronics to similarly coordinate image capture with scale recordings, with the trigger to the CMM being sent from an output signal incorporated into the camera circuitry. The primary concerns for this initial sensor system were light weight, due to the torque limitations of the PH10M probe head, and adjustability of all dimensions to be able to find the optimal camera positions.

The stereo vision sensor was made primarily from aluminum for light weight and high strength, with Delrin plastic being used for some components for the combination of its light weight and its insulating properties. The Delrin also separated the cameras from the CMM electrically. All dimensions of the system can be adjusted, including the distance between camera lenses and their relative angles in both axes. The sensor system attaches to the PAA1 adaptor with an M8 threaded rod, which is electrically isolated from an insert centered within the rod to be able to control the capture signal sent to the CMM. A photograph of the sensor, mounted on the CMM, is shown in Figure 11.

\section{Image Processing}

\section{Camera Calibration}

The camera calibration procedure is based on Zhang [9]. A $30 \mathrm{~mm} \times 20 \mathrm{~mm}$ reference plane with a near perfect $2.54 \mathrm{~mm}$ pitch lithographically produced grid was used. The grid plane was oriented to 9 different positions, and was simultaneously imaged by both cameras. The grid line intersections (vertices) were detected and used to determine two Homogeneous Transformation Matrices (HTMs) that relate each Camera Coordinate Systems (CCS) to an overall sensor Stereo Coordinate System (SCS).

The cameras are translated by CMM axis motion, and rotated using the PH10 pan and tilt capability. Hence, the SCS point coordinates must be translated yet again into a Global Coordinate System (GCS) using registration. For any pair of PH10 poses, the registration transformation that accomplishes this consists of the rotation matrix $\mathbf{R}_{3 \times 3}$ and translation vector $\mathbf{T}_{3 \times 3}$ that minimizes

$$
e^{2}(\mathbf{R}, \mathbf{T})=\frac{1}{n} \sum_{i=1}^{n}\left\|\mathbf{y}_{i}-\left(\mathbf{R} \mathbf{x}_{i}+\mathbf{T}\right)\right\|^{2}
$$

where \|\| is the usual Euclidean distance, the $\mathbf{x}_{i, 3 \times 1}$ are the point coordinates in one pose, and the $\mathbf{y}_{i, 3 \times 1}$ are the coordinates of the corresponding points in the second pose. A closed form solution using the Singular Value Decomposition (SVD) has been reported in [10]. Pennec's iterative mean shape method [11] was used to minimize the accumulated error for $N>2$ poses. Laboratory calibration was carried out using a $150 \mathrm{~mm}$ diameter dome shaped part having the same lithographically produced grid described above.

\section{Grid Intersection Detection}

Grid intersection detection for the stereo CCD camera solution was implemented in 4 sequential steps: 1) noise reduction and gradient image calculation; 2) region segmentation and merging; 3) boundary processing; and 4) 3-D reconstruction. The procedure is illustrated using the original picture shown in Fig. 12.

The first image processing step was to remove high frequency noise using a Gaussian filter [12]. To emphasize the grid edges, a $3 \times 3$ Sobel mask is then used. 


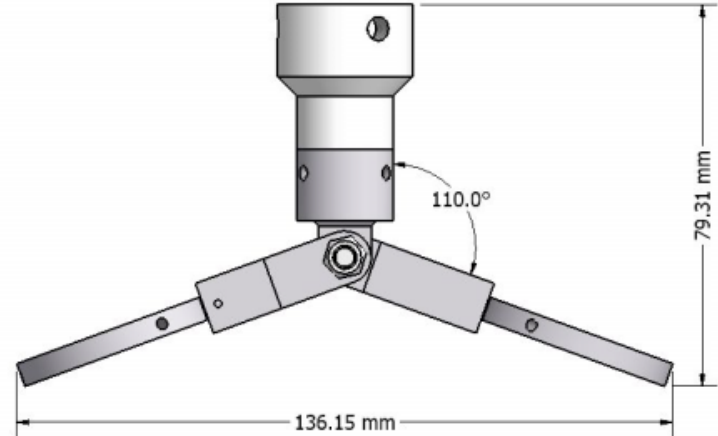

Figure 10 (a). Stereo vision sensor geometry.

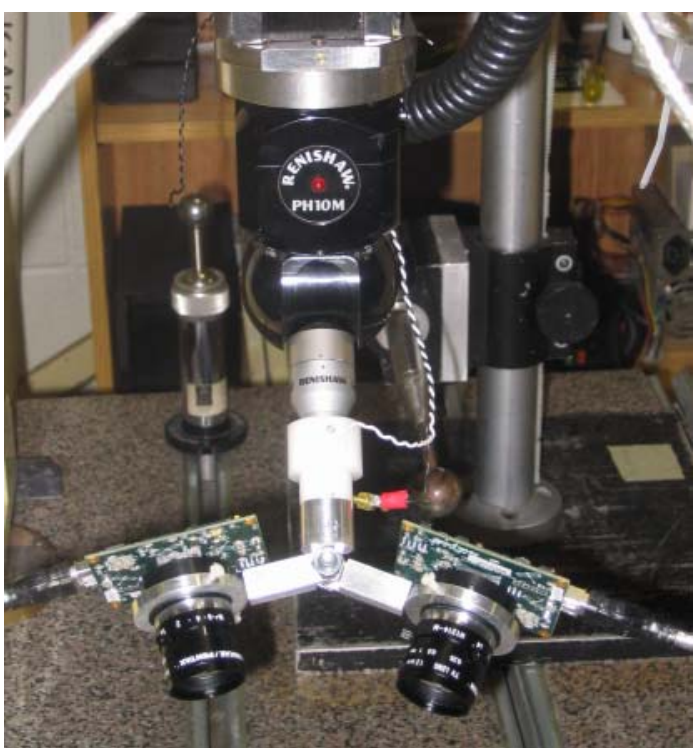

Figure 10 (b). Stereo vision sensor.

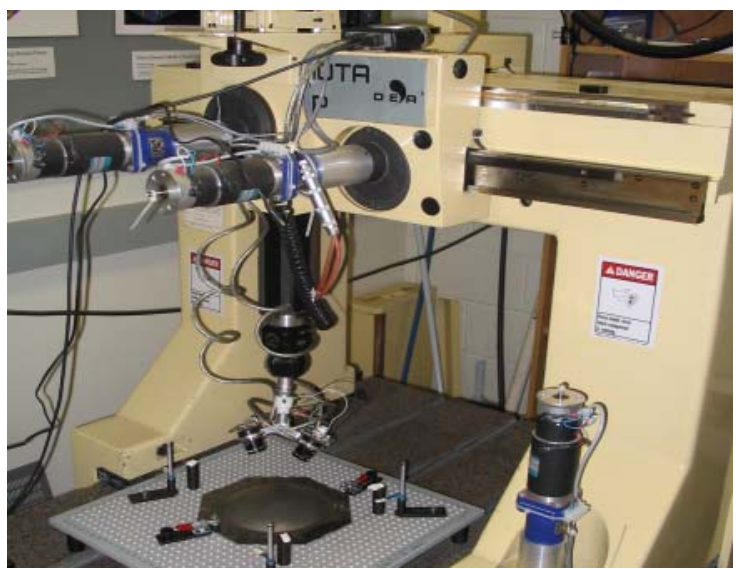

Figure 11. Stereo vision sensor installed on CMM probe head

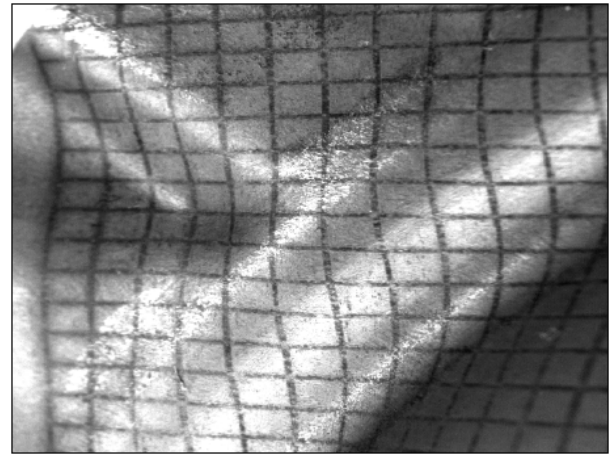

Figure 12. Image processing original picture.

To detect the grid intersection vertices, the images must be segmented into separate regions corresponding to each individual grid element. This task was accomplished using the circular disk dilation [13] method. The method begins by removing small $(<300$ pixel), noisy areas (Fig. 13(a)), and then dilating the remaining boundaries outward using a circular disk (radius 15 pixels) (Fig. 13(b)). Circular disk erosion (radius 15 pixels) is then performed to restore the original boundaries, but with the effect of filling in small missing portions (Fig. 13(c)). Black/white complementing is then performed, and regions that are too small $(<2000$ pixels $)$ or too large (>6500 pixels) are eliminated (Fig. 13(d)).

After merging, the region boundaries are traversed with the highest curvature (k-curvature) being used to locate the 4 boundary points [12] (Fig. 13(e)). The centerline of the edge between regions, and between boundary points, is then computed using the Chamfer 3-4 distance metric [14] (Fig. 13(f)).

Finally, the vertices of 4 adjacent regions are used to define a local coordinate system, and parabolas are then used to refine the vertex intersection coordinates to sub-pixel accuracy (Fig. 13(g,h)).

After all of the above 2-D steps have been completed, vertices in the left and right images are rectified [15] to reduce the matching search space to one dimension, and then matched using triangulation to obtain the corresponding 3-D SCS vertex.

\section{Strain Analysis}

Strain analysis for the square grid can then be carried out using the procedure described in [16]. Briefly, each square is divided into 2 separate triangles, with a local coordinate system origin established at the middle vertex. On the undeformed sheet, the edge vertices will be located at coordinates $\left(X_{1}, Y_{1}\right)$ and $\left(X_{2}, Y_{2}\right)$. After forming, the middle vertex is maintained at the origin, and, applying a constant, 


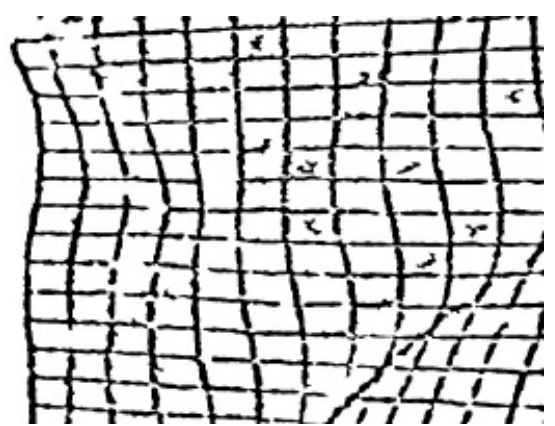

Figure 13(a). Stereo Vision image after removal of noisy areas.

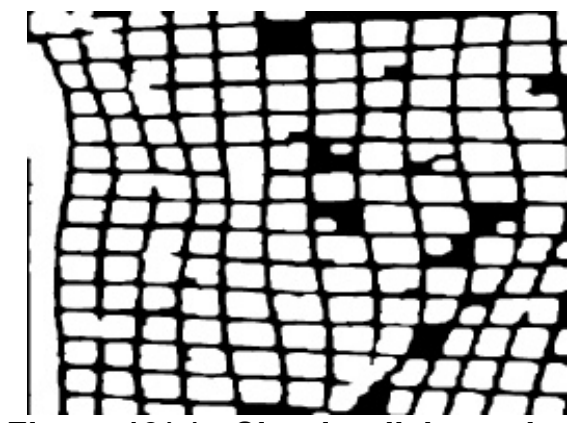

Figure 13(c). Circular disk erosion.

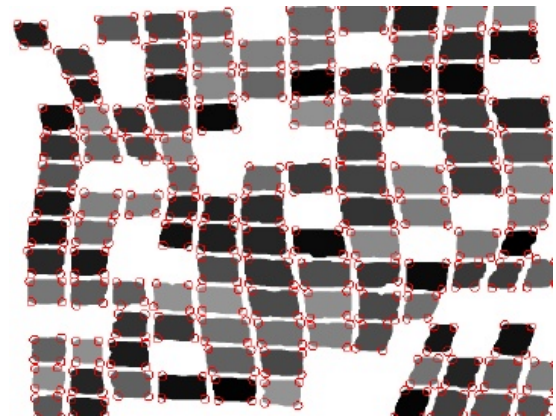

Figure 13(e). Corner detection using kcurvature [12].

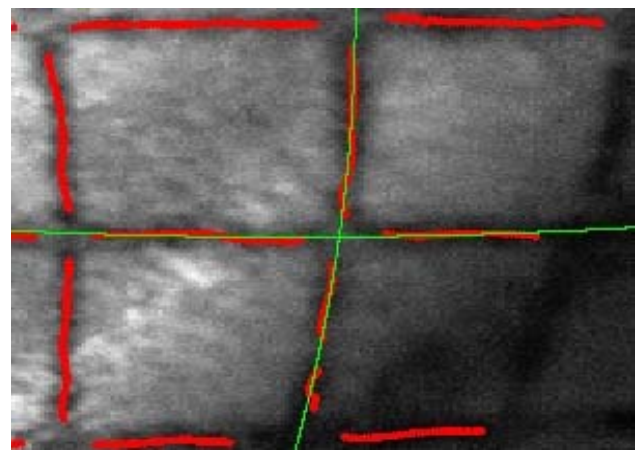

Figure 13(g). Parabolic construction and intersection.

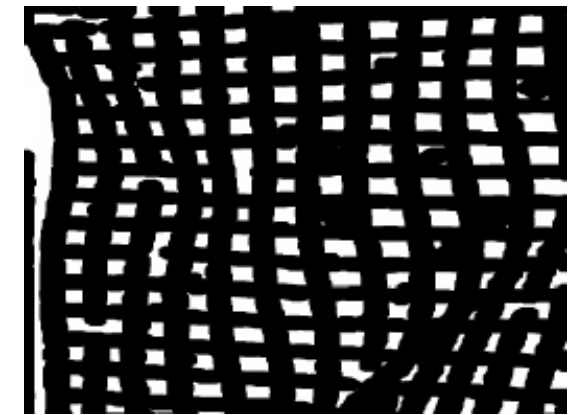

Figure 13(b). Circular disk dilation.

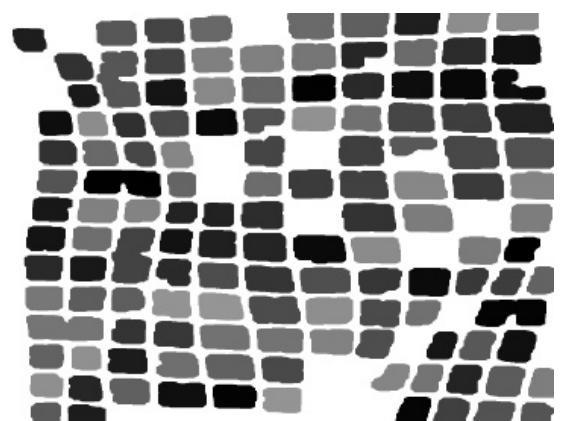

Figure 13(d). Black/white complementing and removal of too small/too large regions.

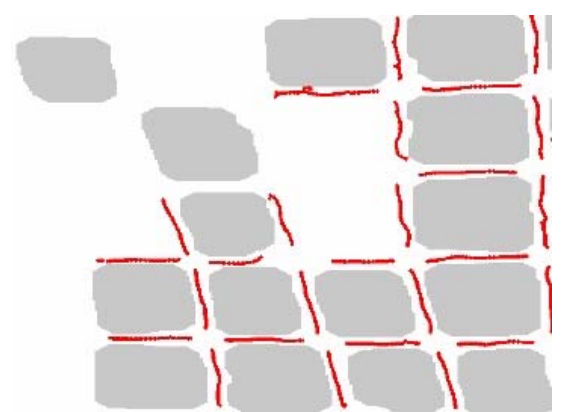

Figure 13(f). Centerline calculation [14].

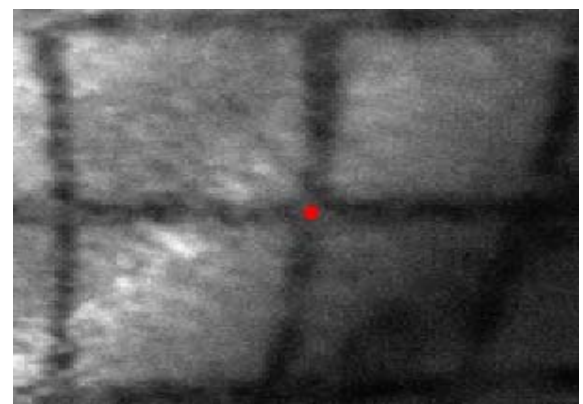

Figure 13(h). Extracted intersection point. 


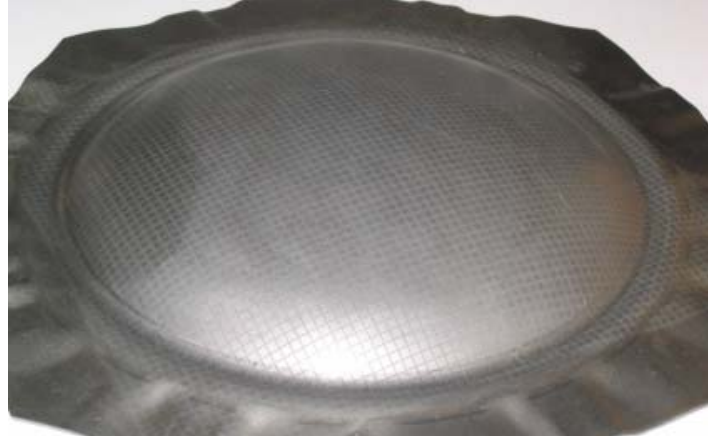

Figure 14(a). Steel test dome.

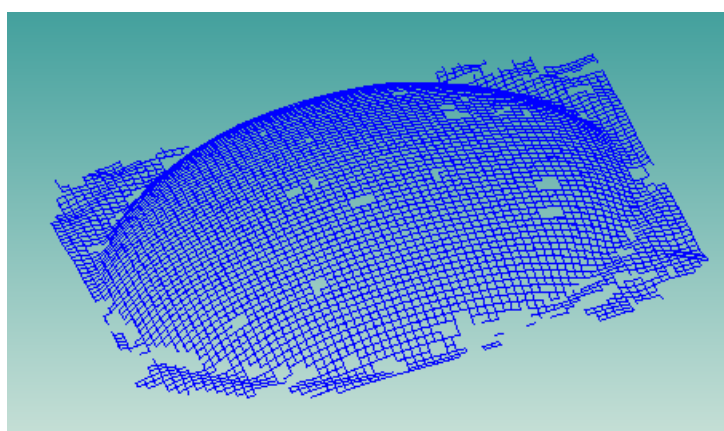

Figure 14(c). Steel dome 3-D HOOPS reconstruction.

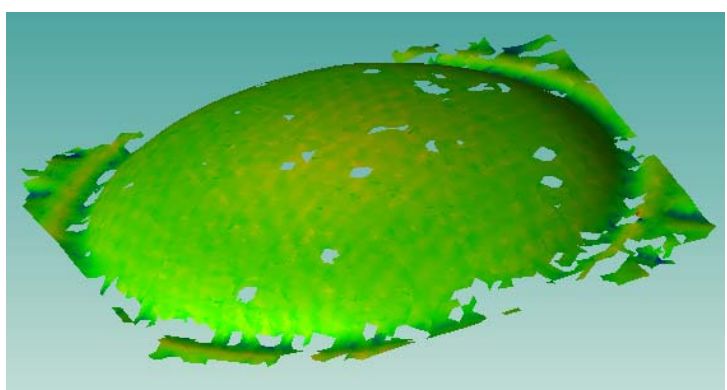

Figure 14(e). Steel dome 3-D HOOPS thickness strain plot.

second order tensor $\mathbf{F}_{2 \times 2}$ to the edge vertices results in the linear systems:

$\left[\begin{array}{l}x_{1} \\ y_{1}\end{array}\right]=\left[\begin{array}{ll}F_{11} & F_{12} \\ F_{21} & F_{22}\end{array}\right] \cdot\left[\begin{array}{l}X_{1} \\ Y_{1}\end{array}\right] ;\left[\begin{array}{l}x_{2} \\ y_{2}\end{array}\right]=\left[\begin{array}{ll}F_{11} & F_{12} \\ F_{21} & F_{22}\end{array}\right] \cdot\left[\begin{array}{c}X_{2} \\ Y_{2}\end{array}\right](4)$

where $\left(x_{1}, y_{1}\right)$ and $\left(x_{2}, y_{2}\right)$ are the deformed edge vertex coordinates. Rearranging,

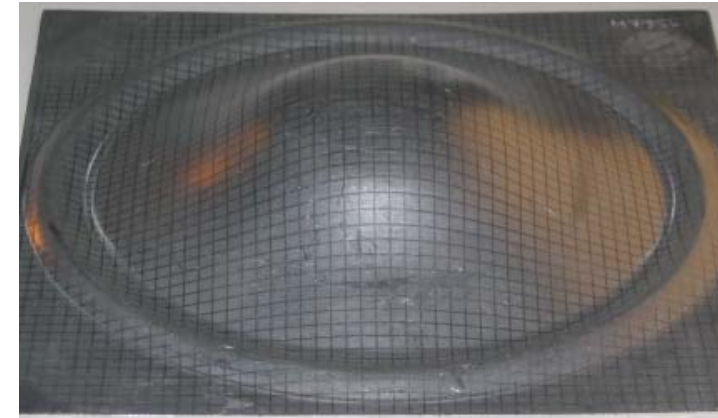

Figure 14(b). Aluminum test dome.

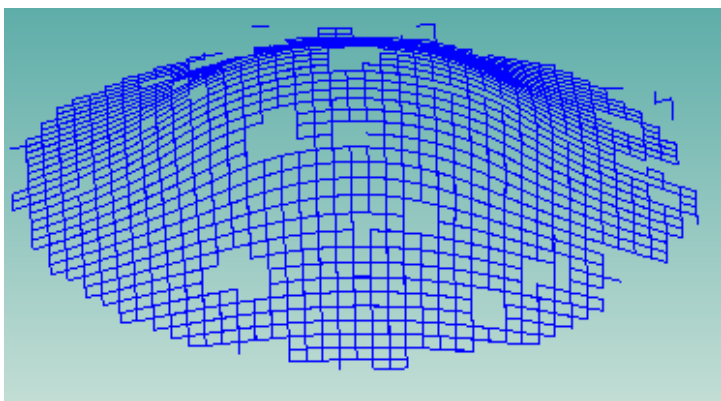

Figure 14(d). Aluminum dome 3-D HOOPS reconstruction.

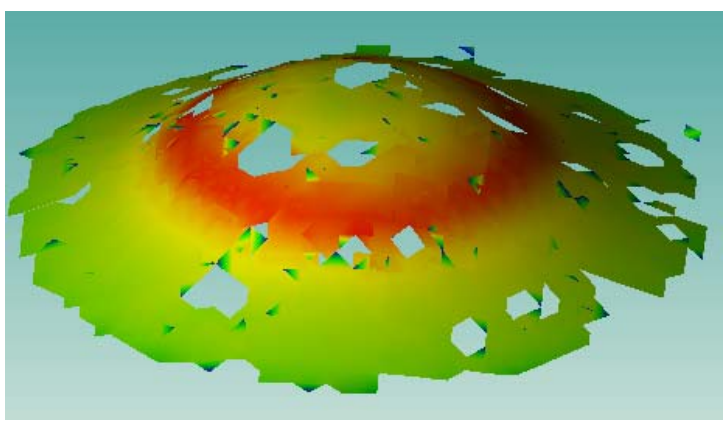

Figure 14(f). Aluminum dome 3-D HOOPS thickness strain plot.

$$
\left[\begin{array}{l}
x_{1} \\
x_{2} \\
y_{1} \\
y_{2}
\end{array}\right]=\left[\begin{array}{cccc}
X_{1} & Y_{1} & 0 & 0 \\
X_{2} & Y_{2} & 0 & 0 \\
0 & 0 & X_{1} & Y_{1} \\
0 & 0 & X_{2} & Y_{2}
\end{array}\right] \cdot\left[\begin{array}{l}
F_{11} \\
F_{12} \\
F_{21} \\
F_{22}
\end{array}\right]
$$

can now be solved for $\mathbf{F}$. The eigenvalues $\lambda_{1,2}$ of $\mathbf{F}$ can be computed using the Singular Value Decomposition (SVD), and satisfy the relationship 


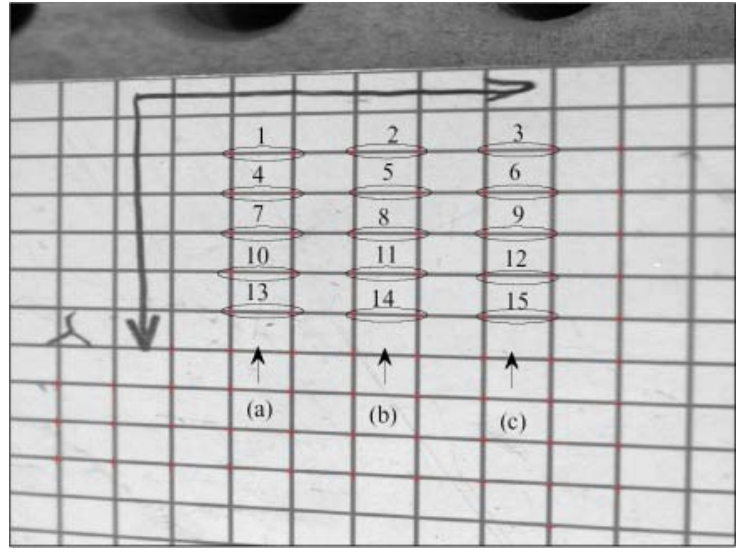

Figure 15(a). Uncertainty analysis test grid.

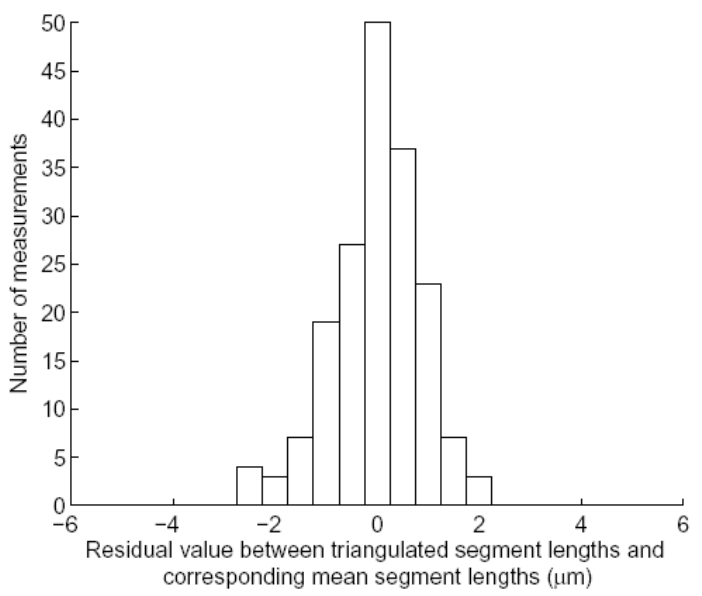

Figure 15(b). Single view uncertainty analysis line segment length histogram.

$\varepsilon_{1,2}=\ln \left(\lambda_{1,2}\right)$ where, as before, $\varepsilon_{1}$ and $\varepsilon_{2}$ are the surface true strains.

\section{HOOPS Visualization}

Manufacturing problems are often of an urgent nature, and therefore it is essential that inspection data be easily interpreted so that corrective action can occur quickly. For this application, the HOOPS 3-D Application Framework [19] was used. During stereo matching, grid vertices are automatically inserted into a data structure that links neighbours. Displaying a wire mesh visualization of the $3-\mathrm{D}$ part surface is a simple matter of drawing line segments between vertex neighbours. Colour strain plots are created by joining three vertices to form a solid triangular shell surface. The scalar thickness strain is then rendered using a "temperature plot" interpolation.

\section{Experimental Results}

The stereo CCD camera solution was tested on an electrochemically etched steel test dome, and a screen printed aluminum test dome. In both cases, a $2.54 \mathrm{~mm}$ pitch square grid was applied before forming.

For the steel test dome (Fig. 14(a)), 25 orientations of the PH10 probe head were used, and 170 separate stereo views of the part were captured. Using an Intel $1.6 \mathrm{GHz}$ computer, 49 hours was required for image processing. Over half of the time was spent on iterative orthogonal least square parabola fitting, and this is due to the very high resolution of pixels. A 3-D plot of the reconstructed grid, containing approximately 6,000 matched vertices, is shown in Fig. 14(c). After global registration, the maximum distance between corresponding points was less than $20 \mu \mathrm{m}$. Less than one minute of additional computer processing time was required for the strain analysis step, and the plotted results are shown in Fig. 14(e).

For the aluminum sheet (Fig. 14(b)), 70 stereo views were captured, and image processing time was 19 hours. A 3-D plot is shown in Fig. 14(d). The strain plot is shown in Fig. 14(f).

\section{Discussion}

Uncertainty estimates for competing systems are summarized in Table 1. For comparison with competing methods, an estimate of the uncertainty of the stereo vision system is a key metric. To investigate this, a precise grid was printed onto a flat aluminum newspaper printing sheet (Fig. 15(a)). The sheet was imaged from 12 different poses, and the lengths of the 15 circled line segments were calculated using the stereo camera algorithms, and compared to $0.005 \mathrm{~mm}$ resolution optical microscope measurements. A histogram of the stereo lengths with respect to their corresponding means is shown in Fig. 15(b). This distribution is Gaussian, with a two standard deviation $(2 \sigma)$ of $0.0016 \mathrm{~mm}$, and hence the uncertainty for a point in a single view is $0.0016 / \sqrt{2}=0.0011 \mathrm{~mm}$. Including the CMM volumetric errors, the $2 \sigma$ uncertainty is estimated to be $0.017 \mathrm{~mm}$. The nominal strain accuracy for a 2.54 $\mathrm{mm}$ square grid is $0.06 \%$, which is better than the design goal.

As mentioned earlier, the primary disadvantage of using laser digitizers for strain analysis is that they are line acquisition devices, and must be moved at a 


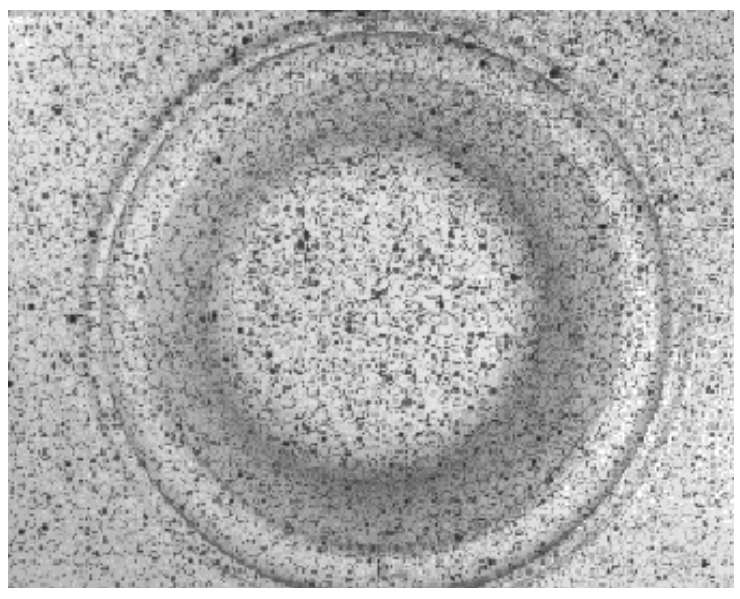

Figure 16. Sheet metal speckle pattern [18].

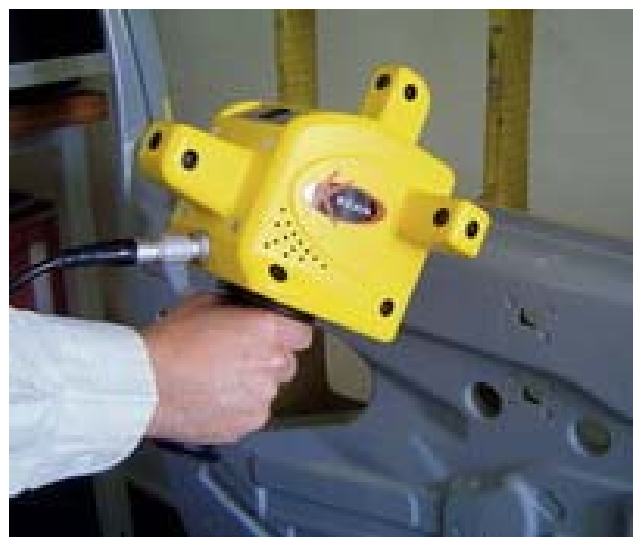

Figure 17. Frameless handheld laser digitizer [17].

constant speed to acquire evenly spaced points. Motorized orthogonal axis CMMs support this, but installation on a FARO arm does not. For these devices, the stereo CCD camera solution, which records an area with each acquisition, is preferred. Multiple line laser digitizers allow more dense data collection, but the grey levels would differ for each beam, making binary thresholding impossible.

With suitable lens choices and a robust mounting enclosure, installation of the stereo vision sensor on the FARO arm or even an industrial robot would be very practical. These devices tend to have larger intrinsic geometric errors, and using the square grid vertex pattern makes it very easy to implement global registration. However, curvature information between the grid vertices is lost. Speckle based methods (Figure 16) [18] offer a solution for this, but require before and after images for correlation. With sufficient accuracy, a frameless handheld unit (Figure 17) [17], with the stereo vision sensors replacing the laser digitizer, has much promise.

\begin{tabular}{|c|c|}
\hline Method & $2 \sigma$ uncertainty \\
\hline Mylar® tape [20] & $10 \%$ \\
handheld camera [3] [20] & $4 \%$ \\
stereo vision [21] & $4 \%$ \\
stereo vision [22] & $5 \%$ \\
laser digitizer [5] & $2.5 \%$ \\
this paper & $0.06 \%$ \\
\hline
\end{tabular}

\section{Table 1. Uncertainty of Strain Analysis Methods.}

The large number of digitized points requires significant data processing time. Much of this can be eliminated by fitting the parabolas to only a fraction of the grid line sample points. For real time display of the area scanned, approximate vertex finding can be used, with more exhaustive analysis being performed offline. Specular reflection from shiny metal surfaces is often an issue. For the work reported herein the steel was galvanized and this was not a significant problem. Polarizing filters, and careful control of ambient lighting, are topics for further research. For geometric digitizing applications other than strain analysis, structured light could also be used instead of etching a grid onto the part.

\section{Conclusions}

This paper has presented the design and implementation of a stereo computer vision sheet metal geometry / strain analysis system suitable for installation on a Coordinate Measuring Machine or portable FARO arm. Accuracy is comparable to other methods, including laser digitizers. Results from test dome measurements demonstrated the system capability, including 3-D graphics visualization. Continuing work is planned to robustly package the sensor, reduce image processing time, and to improve display graphics.

\section{Acknowledgements}

Equipment funding and graduate student stipends for this work were provided by the Natural Sciences and Engineering Research Council of Canada (NSERC). Phil Mitchell performed the stereo image processing work, under the co-supervision of Dr. David Capson of the Department of Electrical and Computer Engineering, McMaster University. The Hamilton Spectator provided the aluminum calibration sheet. 


\section{References}

[1] Keeler, S.P., Training Manual - Enhanced FLC Project, The Auto/Steel Partnership, Southfield, MI, http://www.a-sp.org/.

[2] Dinda, S., James, K.F., Keeler, S.P. and Stine, P.A., How to Use Circle Grid Analysis for Die Tryout, American Society for Metals, Metals Park, OH, 1981.

[3] Forming Measurement Tool Innovations System Inc., Hamilton, ON, CA, www.fmtisystems.com.

[4] Vogel, J.H., and Lee, D., 1990, "The automated measurement of strains from three-dimensional deformed surfaces", Journal of Metals, 43, 1990, pp. 8-13.

[5] Chan, H.-L., Spence, A.D., and Sklad, M.P., "Parallel Computing for Sheet Metal Strain Analysis", HPCS 2005 - 19th Int. Symp. High Performance Computing Systems and Applications, University of Guelph, May 15-18, 2005.

[6] FARO Technologies Inc., Lake Mary, FL, www.faro.com.

[7] Point Grey Research Inc., Vancouver, BC, www.ptgrey.com.

[8] The Imaging Source, Lenses: Optical Basics, Charlotte, NC 28204, www.theimagingsource.com/resources/background/int ro-to-optics_eng.pdf.

[9] Zhang, Z., A Flexible New Technique for Camera Calibration, IEEE Trans. Pattern Analysis And Machine Intelligence, Vol. 22, No. 11, 2000, pp. 13301331.

[10] Umeyama, S., Least-Squares Estimation of Transformation Parameters Between Two Point Patterns, IEEE Trans. Pattern Analysis And Machine Intelligence, Vol. 13, No. 4, 1991, pp. 376-380.

[11] Pennec, X., Multiple registration and mean rigid shapes: Application to the 3D case, 16th Leeds Annual Statistical Workshop, Leeds, UK, 1996, pp. 178-185.

[12] Jain, R., Kasturi, R. and Schunck, G., Machine Vision, McGraw-Hill, New York, NY, 1995.

[13] Gonzalez, R.C. and Woods, R.E., Digital Image processing, Addison-Wesley, Reading, MA, 1992.

[14] Borgefors, G., Distance Transformations in Digital Images, Computer Vision, Graphics and Image Processing, Vol. 34, No. 3, 1986, pp. 344-371.

[15] Fusiello, A., Trucco, E. and Verri, A., A compact algorithm for rectification of stereo pairs, Machine Vision and Applications, Vol. 12, 2000, pp. 16-22.

[16] Sowerby, R., Duncan, J.L. and Chu, E., The Modelling of Sheet Metal Stampings, Int. J. Mechanical Sciences, Vol. A28, No. 7, 1986, pp 415-430.

[17] Metris, Belgium, www.belgium.be.

[18] Garcia, D., and Orteu, J.J., "3D Deformation Measurement using Stereo-Correlation Applied to Experimental Mechanics", The $10^{\text {th }}$ FIG Int. Symp. Deformation Measurements, Orange, CA, 2001, pp. 50-60.

[19] Techsoft America, LLC, Oakland, CA, www.hoops3d.com.
[20] M.P. Sklad, "Aspects of automated measurement of proportional and non-proportional deformation in sheet metal forming", Journal of Materials Processing Technology, 145, 2004, pp. 377-384.

[21] D.W. Manthey, R.M. Bassette, and D. Lee, "Comparison of different surface strain measurement techniques used for stamped sheet metal parts", Proceedings of International Body Engineering Conference: Body Assembly \& Manufacturing, Sep. 21-23, 1993, pp. 106-111.

[22] D.W. Manthey, D. Lee, "Vision based surface strain measurement system", The Journal of the Minerals, Metals, \& Material Society (JOM), 47(7), 1995, pp. 46-49. 